\title{
Continuous Sampling of Immune Cells in the Skin by Dermal Open Flow Microperfusion
}

T. Birngruber ${ }^{1}$, B. Prietl ${ }^{2}$, M. Bodenlenz ${ }^{1}$, P. Florian ${ }^{3}$, A.Subramaniam ${ }^{3}$, S. Kainz ${ }^{1}$, G. Rauter ${ }^{1,4}$, F. Sinner ${ }^{1,2}$

CONTACT

1 JOANNEUM RESEARCH Forschungsgesellschaft mbH

HEALTH

nstitute for Biomedicine and

Health Sciences

Neue Stiftingtalstrasse 2
8010 Graz

Phone $+43316876-4000$

Fax +43 $3168769-4010$

health@joanneum.at

www.joanneum.at/health

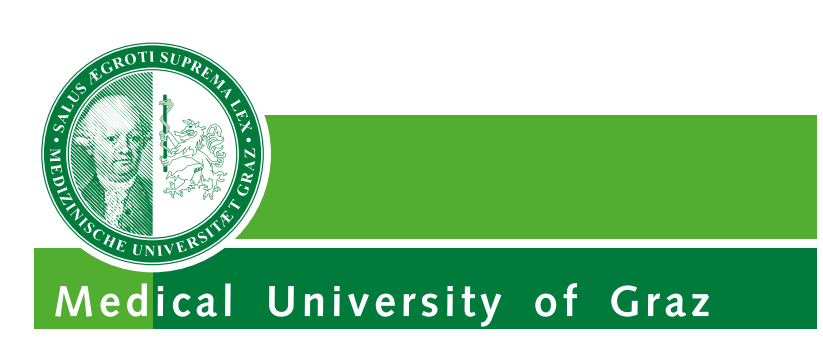

2 Medical University of Graz

Division of Endocrinology and Diabetology

Graz, Austria

\section{SANOFI GENZYME Y}

${ }^{3}$ Sanofi-Genzyme Framingham, MA USA and Sanofi Frankfurt, Germany.

\section{References}

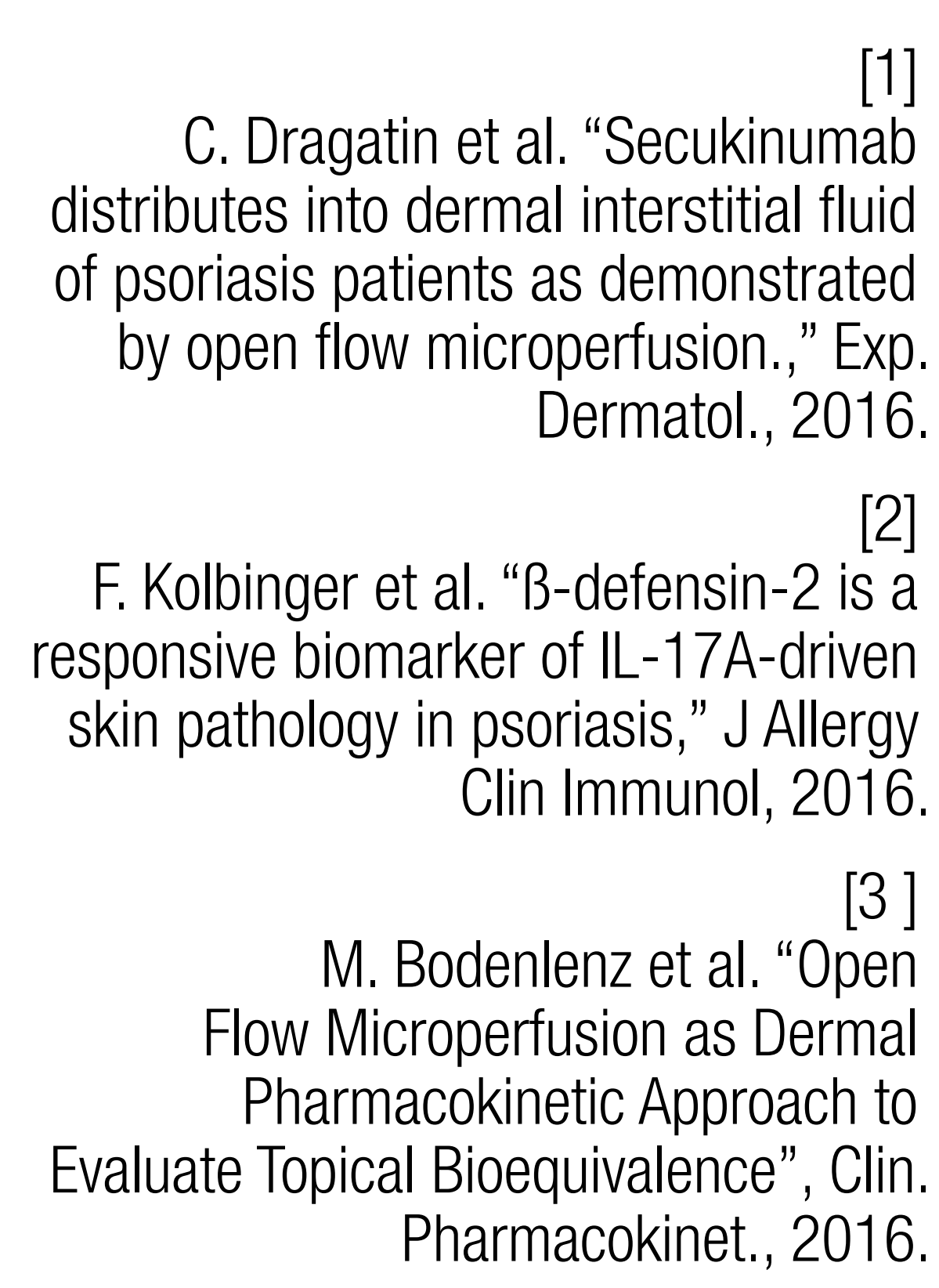

Purpose

Psoriasis vulgaris is an inflammatory skin disease that is based on an autoimmune reaction involving immune competent cells and molecules of both the innate and the adaptive immune system. The boundaries of the inflammatory lesions are clearly delimited which results in different inflammation and immune status inside and outside the lesions. Currently, skin inflammation and immune cell populations are investigated using highly invasive biopsies. We investigated whether the minimally invasive dermal Open Flow Microperfusion (dOFM) enables continuous sampling of immune cells from inflamed and healthy skin in a psoriasis rat model

\section{Methods}

OFM is a minimally invasive probe-based method that is used to evaluate the pharmacokinetics and pharmacodynamics of drugs directly in skin. The use of the OFM probes allows small amounts of interstitial fluid (ISF) to be collected from target tissues for analysis. OFM has been successfully used in for continuously sampling in dermal tissue in preclinical and clinical studies for PK/PD studies as well as topical bioequivalence trials ${ }^{[1-3]}$

Physiological fluid is pumped through the dOFM probes applying a welldefined pump speed. The dOFM probes feature macroscopic openings to allow exchange of substances between the physiological and the interstitial fluid (ISF) in the surrounding tissue. dOFM samples represent diluted but otherwise unchanged ISF samples. This enables sampling of highly lipophilic substances and of substances with high molecular weight, up to entire cells.

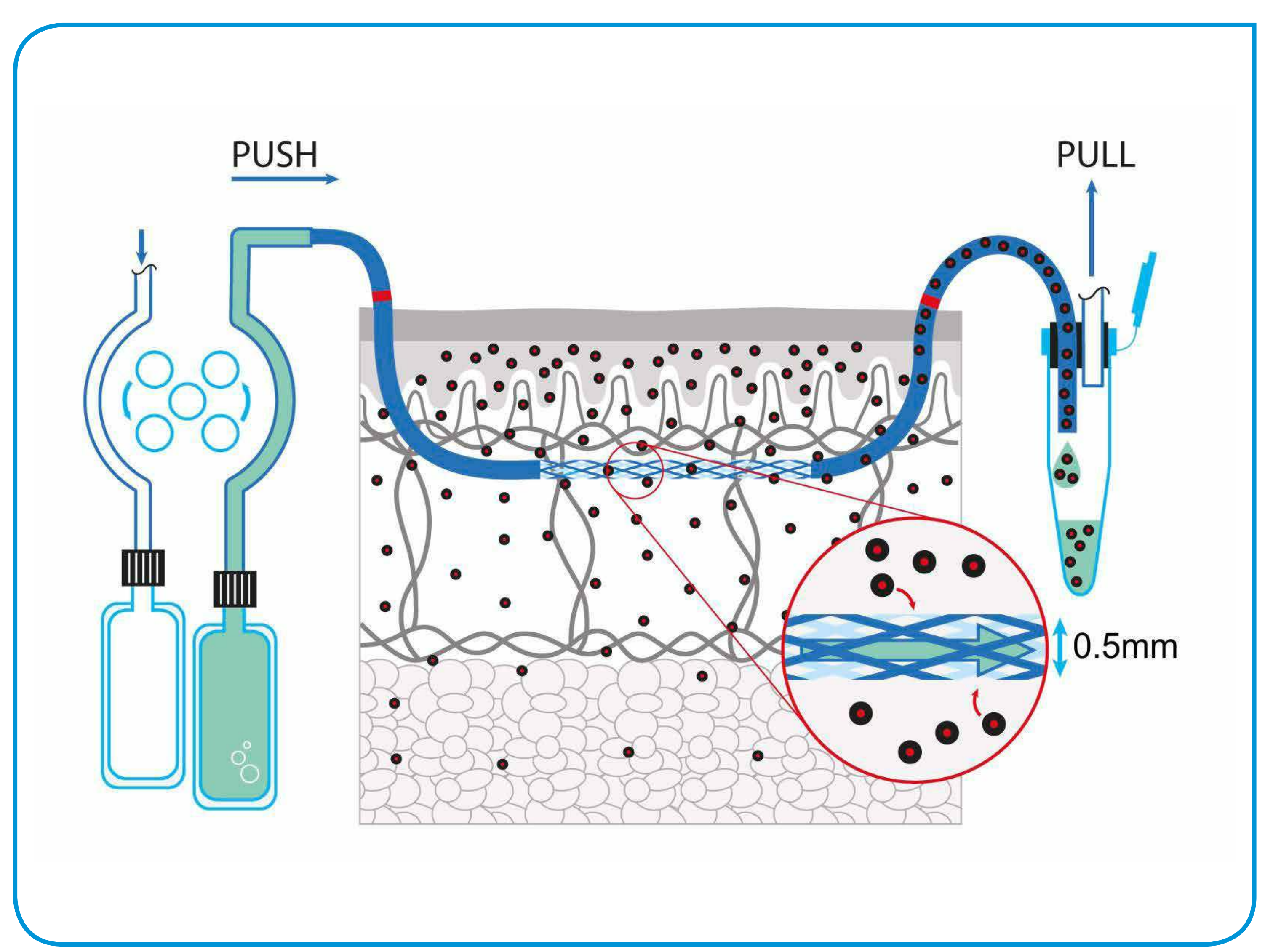

Figure 1: Principle of dOFM sampling. dOFM continuously delivers unfiltered dermal interstitial fluid for analysis of biomarkers, drugs, and immune cells.
In this study we used a rat psoriasis model based on the topical application of imiquimod (IMQ) cream

- Sprague-Dawley rats

- Topical treatment with $I \mathrm{MQ}$ for 8 days on right back

- Untreated control site on left back

- Rats were divided in 2 groups $n=6$ :

$\Rightarrow$ Group (A) received oral placebo daily

- Group (B) received oral dexamethasone daily

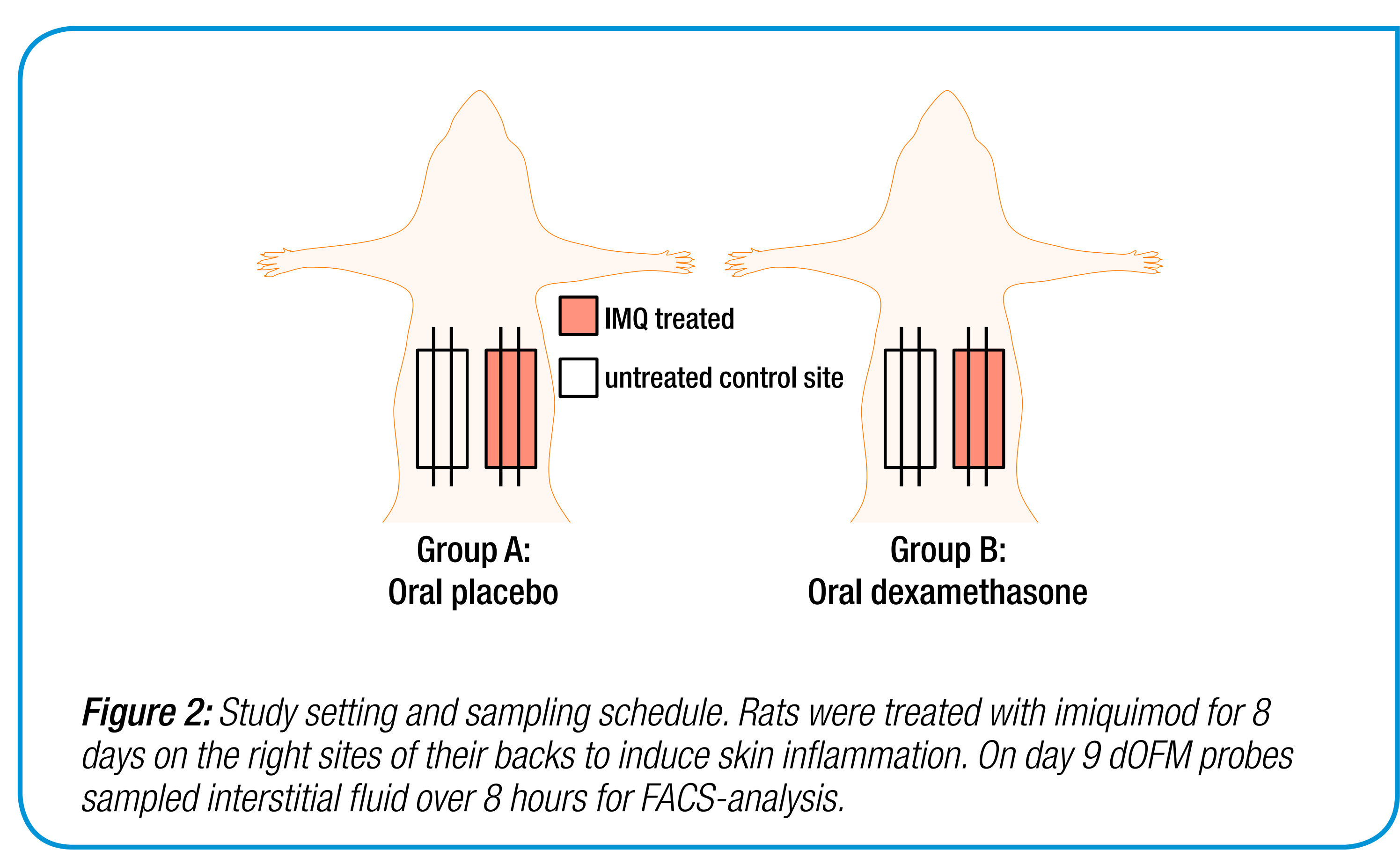

Immune cell analysis by flow cytometry (FACS)

- Staining of cells by monoclonal fluorochrome-labelled antibodies

- Use of lyse-no-wash procedure \& eCount beads (Thermofisher Scientific)

- Quantitative analysis of immune cells using BD TruCOUNT tubes

\section{Results \& Discussion}

Immune cell analysis in dOFM samples revealed (Fig. 3 and 4 )

- IMQ treated skin shows increased levels of granulocytes, CD 8cells, TH17 and IL17+ cells in the oral placebo group (Figure 3)

- Dexamethasone lowered most cell types relevant for inflammation in the skin

- FACS analysis of OFM samples reliably displayed the cell population in the skin. The findings were confirmed by proteomics and visual evaluation of the inflamed skin (see poster \# 502)

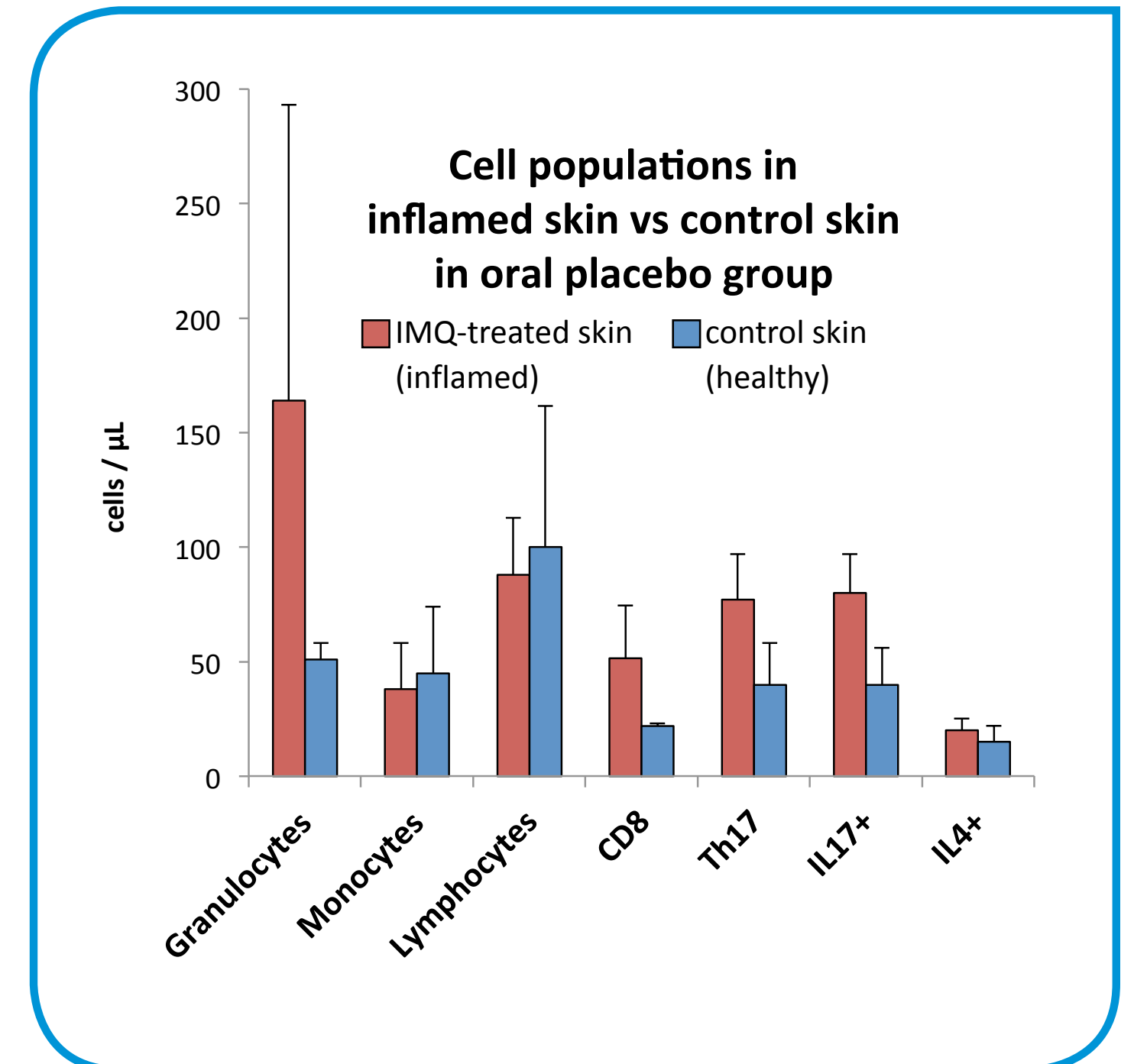

Figure 3: Major immune cell classes (granulocytes, monocytes, lymphocytes) and T-cell subtypes (CD8, Th17, lL17+, lL4+) in dermal OFM samples. Red columns represent OFM samples from inflamed skin and blue columns represent OFM samples from healthy control skin of the same rats.

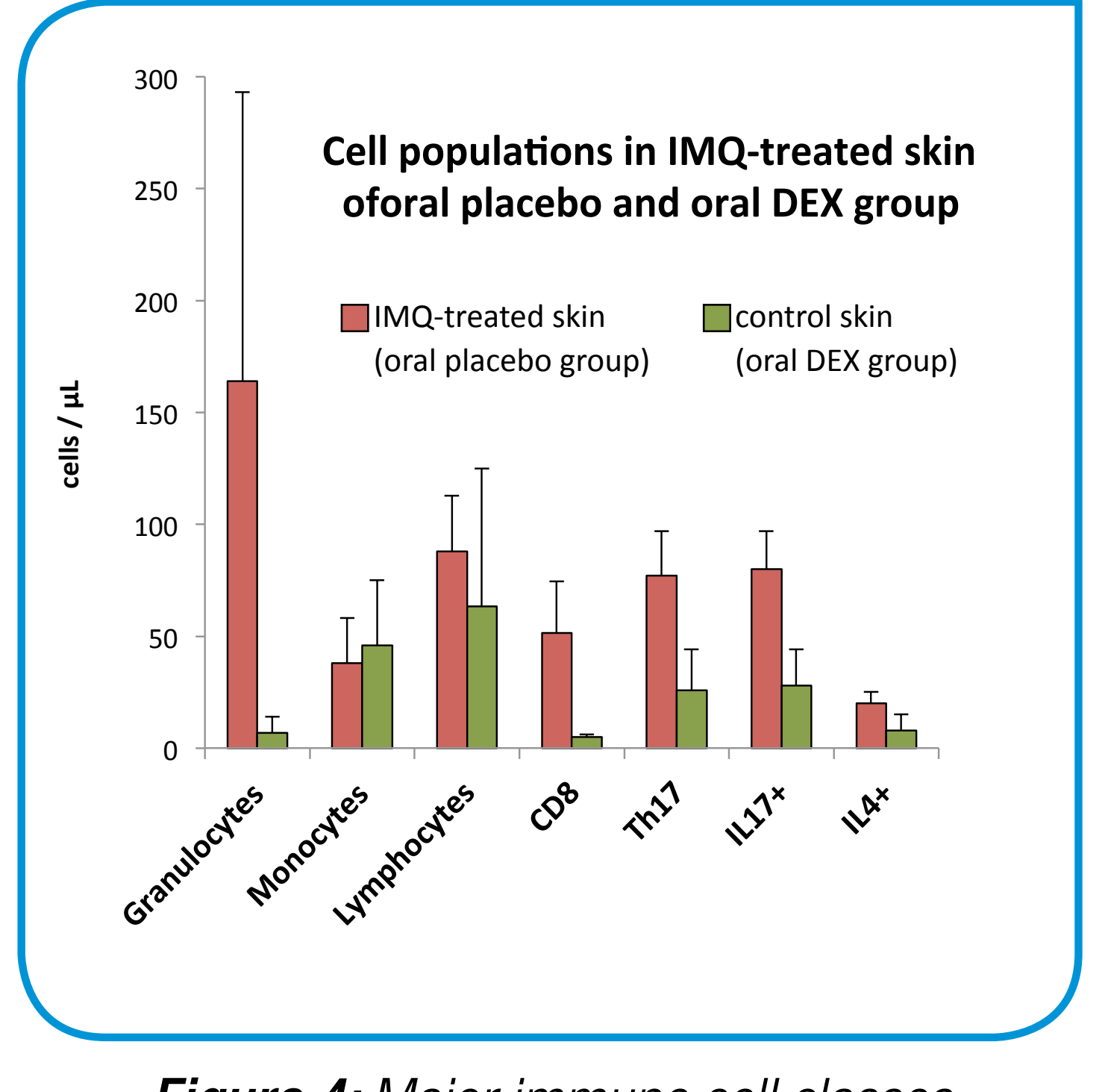

Figure 4: Major immune cell classes (granulocytes, monocytes, lymphocytes) and T-cell subtypes (CD8, Th17, LL17+, LL4+) in dermal OFM samples. All OFM samples are from skin with IMQ treatment, rats that received additional dexamethasone (DEX) treatment are represented in the green columns.
Conclusions

dOFM enables the continuous sampling of immune-competent cells from living skin. The resulting measured cell population allows differentiation of healthy and inflamed skin and identification of the treatment effect of dexamethasone.

- Results are in line with results from proteomics and applied standard psoriasis scores.

- Results from this study enable investigation of autoimmune diseases in a novel, innovative way, especially when performing target identification in drug research. 